\title{
Preservation of properties in nonstandard hulls of $C^{*}$-algebras
}

\author{
Stefano Baratella
}

\begin{abstract}
We extend the nonstandard hull construction of Luxemburg and we show that the proposed extension covers various other constructions such as the tracial and the weak nonstandard hull. We provide a novel proof that the tracial nonstandard hull of an internal von Neumann algebra is itself a von Neumann algebra. We prove that some extensively studied properties of $C^{*}$-algebras are preserved and reflected by the nonstandard hull construction. Eventually, we show how to obtain hull-like representations of the two $K$-groups associated to the nonstandard hull of an internal $C^{*}$-algebra.
\end{abstract}

\section{Introduction}

In this paper we first provide a unifying approach to different constructions that turn out to be generalizations of the nonstandard hull construction due to Luxemburg [19]. It is well known that, in turn, the nonstandard hulls generalize the ultraproducts introduced in the framework of functional analysis by Dacunha-Castelle and Krivine [10]. (See the remarks in [15].)

In Section 2 we fix some notation and we recall the main tools which are available in a nonstandard setting. In this section we also try to convey some basic ideas about the use of nonstandard techniques à la A. Robinson.

For sake of completeness, in Section 3 we present the original nonstandard hull construction. We generalize this construction in Section 4. In spite of its simplicity, the proposed generalization is powerful enough to cover the tracial and

Received by the editors in April 2013 - In revised form in July 2014.

Communicated by F. Point.

2010 Mathematics Subject Classification : Primary: 46S20. Secondary: 03H05 26E35 46L05.

Key words and phrases : nonstandard hull, $C^{*}$-algebra. 
the weak nonstandard hull constructions, as shown in Subsections 4.1 and 4.2 respectively. In Subsection 4.1 we also provide a proof that the tracial nonstandard hull of an internal von Neumann algebra is itself a von Neumann algebra.

In section 5 we show that the nonstandard hull of a factor in the class of internal weakly central $C^{*}$-algebras is itself a factor $C^{*}$-algebra. We also prove that, for an internal $C^{*}$-algebra, the properties of being prime and primitive are preserved and reflected by the nonstandard hull construction.

In section 6, we provide "hull-like" representations of the $K_{0}$ - and the $K_{1}$-groups associated to the nonstandard hull of an internal $C^{*}$-algebra. This is not completely trivial because, speaking in terms of ultraproducts, in general the $K_{0}$-group of an ultraproduct of $C^{*}$-algebras is not the model theoretic ultraproducts of the $K_{0}$-groups of the factors. Similarly for the $K_{1}$-group. Actually, in order to give a hull-like representation of the $K_{1}$-group of a nonstandard hull, we resort to the original definition in terms of the notion of suspension.

In the attempt to make the paper potentially interesting to both logicians and functional analysts, we often recall basic facts about nonstandard techniques or operator theory. For the same reason, some proofs do contain more details than actually needed.

In the following the reader will find some very specific comments that are mostly intended either for the logicians or for the functional analysts. These comments can be safely skipped by the non-experts in the field they pertain to.

A final comment: the results presented in this paper can also be obtained in the setting of ultraproducts of normed structures and, very likely, in variants of the continuous logic setting introduced in [4]. The choice of nonstandard hulls is just a matter of the author's familiarity with nonstandard techniques.

\section{Preliminaries}

We assume familiarity with the basics of Banach space theory and of the theory of operator algebras, as introduced, for instance, in [22] and [5] respectively.

We also assume familiarity with the construction of a nonstandard universe, and with the nonstandard techniques, as presented in [6], [1] or [24]. In the following we briefly introduce the notion of nonstandard universe and the tools that are available therein. We refer the reader to the suggested bibliography for the technical details.

Nonstandard universe. A nonstandard universe is a set-theoretic construction that allows to properly extend each infinite mathematical object $A$ under consideration to an object ${ }^{*} A$, in a way that $A$ and ${ }^{*} A$ satisfy the same first order properties that are expressible by means of bounded quantifier formulas in the language of set theory. This is referred to as the Transfer Principle. More precisely, one starts with an infinite set $X$ of individuals (to be regarded as ur-elements) and closes it under countably many applications of union and power set operation. The limit $V(X)$ is called the superstructure constructed on $X$. Then, by means of a bounded ultrapower construction (see [6]), one gets: 
1. an infinite set $Y$ (usually a nontrivial ultrapower of $X$ );

2. the structure $(V(Y), \in)$, where $V(Y)$ is the superstructure constructed on $Y$;

3. a bounded elementary embedding ${ }^{*}:(V(X), \in) \rightarrow(V(Y), \in)$ with the properties that ${ }^{*} X=Y$ and, for every infinite set $A \subseteq V(X)$, the set $\left\{{ }^{*} a: a \in A\right\}$ is properly contained in ${ }^{*} A$.

We recall that the property of elementary embedding means that, for each bounded quantifier formula $\varphi\left(x_{1}, \ldots, x_{n}\right)$ in the language of set theory and each $A_{1}, \ldots, A_{n} \in V(X)$, the following are equivalent:

1. $\varphi$ is true of $A_{1}, \ldots, A_{n}$ in $(V(X), \in)$;

2. $\varphi$ is true of ${ }^{*} A_{1}, \ldots,{ }^{*} A_{n}$ in $\left(V\left({ }^{*} X\right), \in\right)$.

Borrowing the model-theoretic notation, we write the equivalence of (1) and (2) as follows:

$$
(V(X), \in) \models \varphi\left[A_{1}, \ldots, A_{n}\right] \Leftrightarrow\left(V\left({ }^{*} X\right), \in\right) \models \varphi\left[{ }^{*} A_{1}, \ldots,{ }^{*} A_{n}\right] .
$$

The triple $\left(V(X), V\left({ }^{*} X\right),{ }^{*}\right)$ is called a nonstandard universe. When there is no ambiguity, one simply says that $V\left({ }^{*} X\right)$ is a nonstandard universe. If $A \in$ $V(X)$, the set ${ }^{*} A$ is called the nonstandard extension of $A$.

In the rest of this section we fix a nonstandard universe $\left(V(X), V\left({ }^{*} X\right),{ }^{*}\right)$ and we introduce its main features.

Transfer Principle. As previously hinted, a bounded elementary embedding is an elementary embedding with respect to the class of bounded quantifier formulas in the language of set theory. The Transfer Principle is just the statement that the * map is a bounded elementary embedding. One use of the Transfer Principle is the following: suppose we want to prove a property of some object $A \in V(X)$ and suppose that this property can be expressed by means of a bounded quantifier formula $\varphi(x)$ in the language of set theory. We try to prove instead that $\varphi\left[{ }^{*} A\right]$ holds in $\left(V\left({ }^{*} X\right), \in\right)$, which might be easier for the reason that $\left(V\left({ }^{*} X\right), \in\right)$ is usually a much richer structure than $(V(X), \in$ ) (see the paragraph about Saturation below). If we succeed, we "transfer" back and we get that $\varphi[A]$ holds in $(V(X), \in)$.

Standard and internal elements. In $V\left({ }^{*} X\right)$, there are standard, internal and external elements. Standard elements are those belonging to the range of the ${ }^{*}$ map. An internal element is a member of some standard sets. External means non-internal.

Saturation. Let $\kappa$ be an uncountable cardinal. A nonstandard universe is $\kappa$-saturated if every family $F$ of fewer than $\kappa$ internal sets that has the finite intersection property (i.e. $\bigcap G \neq \varnothing$ for any finite subfamily $G$ of $F$ ) satisfies $\bigcap F \neq \varnothing$. So $\kappa$-saturation is a $\kappa$-compactness property for the family of internal sets: it ensures enough set existence. The existence of nonstandard universes of any prescribed degree of saturation follows from the results in [6, §5.1].

Internal Definition Principle. Since the Transfer Principle applies to bounded formulas, it refers to internal elements. For instance, assuming that $\mathbb{N} \subset X$, the 
"transfer" of the well-ordering property of $(\mathbb{N},<)$ is the statement that every nonempty internal subset of ${ }^{*} \mathbb{N}$ has least element with respect to the nonstandard extension of $<$. The Saturation property also refers to (families of) internal sets. Hence it is important to establish sufficient conditions for being an internal set. This is the aim of the Internal Definition Principle of Keisler ([6, §4.4]). It states that if $\varphi\left(x_{1}, \ldots, x_{n}, y\right)$ is a bounded quantifier formula and $A_{1}, \ldots, A_{n}, B$ are internal sets, then the set defined in $\left(V\left({ }^{*} X\right), \in\right)$ by the formula with parameters $\varphi\left(A_{1}, \ldots, A_{n}, y\right)$, denoted by

$$
\left\{y \in B:\left(V\left({ }^{*} X\right), \in\right) \models \varphi\left[A_{1}, \ldots, A_{n}, y\right]\right\},
$$

is internal.

In practice, when using nonstandard techniques, one does not need to specify a nonstandard universe. Similarly to a monster model in model theory, one assumes the existence of a sufficiently saturated nonstandard universe $(V(X)$, $\left.V\left({ }^{*} X\right),{ }^{*}\right)$ such that $V(X)$ contains all the ordinary mathematical objects under consideration.

Actually, since a significant amount of functional analysis can be developed inside $V(\mathbb{R})$, we can assume that we work in a sufficiently saturated nonstandard universe $\left(V(\mathbb{R}), V\left({ }^{*} \mathbb{R}\right),{ }^{*}\right)$, where ${ }^{*} \mathbb{R}$ is some nontrivial ultrapower of the reals and the restriction of the ${ }^{*}$ map to $\mathbb{R}$ is just the identity. In particular we have $\mathbb{N} \subset{ }^{*} \mathbb{N}$ and, by the Transfer Principle, ${ }^{*} \mathbb{N} \backslash \mathbb{N}$ is an external set (see the beginning of paragraph on the Internal Definition Principle). This shows why the * map cannot be an elementary embedding with respect to the class of all formulas.

In the following, we always work under the assumption of "sufficient saturation", $\omega_{1}$-saturation being the minimum requirement.

A remark on the terminology: in the following we shall use the attribute "ordinary" for the usual mathematical objects. Typically they will be structures arising in functional analysis. We would rather use "standard" for "ordinary", if it were not the case that "standard" has the precise technical meaning explained above.

So if $\mathcal{X} \in\left\{\right.$ semi-normed, Banach, $C^{*}$-, von Neumann $\}$, an "ordinary $\mathcal{X}$ algebra" will be just a " $\mathcal{X}$ algebra" in the usual sense.

An "internal $\mathcal{X}$ algebra" will be a structure which lives in some nonstandard universe and satisfies the transfer of all the properties defining an ordinary $\mathcal{X}$ algebra. In particular, since we stipulate that the ordinary $\mathcal{X}$ algebras are complex algebras, the internal $\mathcal{X}$ algebras are algebras on some nonstandard extension ${ }^{*} \mathrm{C}$ of the complex field.

Ordinary (internal) $C^{*}$-algebras are always unital. We denote by 1 the algebra unit and we often identify $\mathbb{C} 1\left({ }^{*} \mathbb{C} 1\right)$ with $\mathbb{C}\left({ }^{*} \mathbb{C}\right)$.

A comment on the use of the * symbol: to avoid confusion, the reader should keep in mind that $\mathrm{a}^{*}$ on the left-hand side of an ordinary mathematical object refers to its nonstandard extension. A* on the right-hand side of an object (ordinary or internal) refers to the adjoint of that object, whenever that makes sense. In our opinion, the use of two different star symbols would not provide any 
significant simplification. Worse than that, it would possibly make either the nonstandard or the operator theory people uncomfortable.

We fix some notation and further nonstandard terminology. Let $\mathcal{M}$ be an internal $\mathcal{X}$ algebra with (semi-)norm \|\| . Let $u, v \in \mathcal{M}$. We write $u \approx v$ if $\|u-v\|<\frac{1}{n}$ for all $0<n \in \mathbb{N}$. In such case we say that $u$ and $v$ are infinitely close. If $\|u\|<n$ for some $n \in \mathbb{N}$, we say that $u$ is finite. We denote by $\operatorname{Fin}(\mathcal{M})$ the set of finite elements of $\mathcal{M}$ and by $I$ the set of infinitesimal elements, namely $I=\{x \in \mathcal{M}:\|x\| \approx 0\}$.

For $r \in \operatorname{Fin}\left({ }^{*} \mathbb{R}\right)$ it can be shown that there exists a unique $s \in \mathbb{R}$ such that $r \approx s: s$ is called the standard part of $r$ and is denoted by ${ }^{\circ} s$.

By an ideal in a $\mathcal{X}$ algebra, we always mean a closed, two-sided, proper ideal. Hence an ideal in a $C^{*}$-algebra $\mathcal{M}$ is a closed linear subspace of $\mathcal{M}$ which is closed under left and right multiplication by elements of $\mathcal{M}$ and under involution (we often refer to the latter property as to self-adjointness). Actually, for a $C^{*}$-algebra ideal, self-adjointness is a consequence of the other properties (see [5, Proposition II.5.1.1]).

If property $\mathcal{Y}$ is stronger than property $\mathcal{X}$, we may consider $\mathcal{X}$ ideals in a $\mathcal{Y}$ algebra, with an obvious meaning of the terminology.

A homomorphism of $C^{*}$-algebras will always be an algebra homomorphism that commutes with the involution.

We shall make use of the Strong Operator Topology (SOT, for short) on the ordinary $C^{*}$-algebra $\mathcal{B}(\mathcal{H})$ of bounded linear operators on an Hilbert space $\mathcal{H}$. We recall that the SOT is the topology of pointwise convergence on $\mathcal{B}(\mathcal{H})$ : a net $\left\{a_{i}\right\}_{i \in I}$ in $\mathcal{B}(\mathcal{H})$ converges to $a \in \mathcal{B}(\mathcal{H})$ if and only if, for all $v \in \mathcal{H},\left\{a_{i}(v)\right\}_{i \in I}$ converges strongly (i.e. in the norm topology of $\mathcal{H}$ ) to $a(v)$.

The importance of the SOT lies in the celebrated Kaplansky's Bicommutant Theorem stating that the following are equivalent for a unital, self-adjoint subalgebra $\mathcal{M}$ of $\mathcal{B}(\mathcal{H})$ :

1. $\mathcal{M}$ is a von Neumann algebra (i.e. $\mathcal{M}$ is equal to its bicommutant);

2. $\mathcal{M}$ is closed in the SOT.

Further notions and results will be introduced when they are used.

\section{The nonstandard hull}

In this section we review Luxemburg's nonstandard hull construction. We present it in a slightly different manner from Luxemburg's, for sake of generalizing the construction later on.

We shall define the nonstandard hull of an internal $C^{*}$-algebra $\mathcal{M}$. The $C^{*}$-algebra case does cover all the others, with the exception of von Neumann algebras. It is known among the functional analysts that the ultrapower of a $C^{*}$-algebra $\mathcal{N}$ can be faithfully represented as a von Neumann algebra if and only if $\mathcal{N}$ is finite dimensional. Not surprisingly, an analogous result holds for nonstandard hulls: the nonstandard hull of an internal $C^{*}$-algebra $\mathcal{M}$ can be faithfully represented as a von Neumann algebra if and only if $\mathcal{M}$ is (standard) 
finite dimensional. We sketch a proof of the latter result in Section 4.1. In light of that result, in Section 4.1 we shall work with the tracial nonstandard hull, a modified construction that preserves the property of closedness with respect to the Strong Operator Topology (see the Introduction).

Let \|\| be the norm on $\mathcal{M}$. We first notice that $\operatorname{Fin}(\mathcal{M})$ is an ordinary complete semi-normed self-adjoint algebra with respect to the semi-norm ${ }^{\circ}\|\|$ (the standard part of the internal norm). Moreover, $\operatorname{Fin}(\mathcal{M})$ satisfies the $\mathrm{C}^{*}$-condition ${ }^{\circ}\left\|x^{*} x\right\|=\left({ }^{\circ}\|x\|\right)^{2}$. Completeness of ${ }^{\circ}\|\|$ is the only property whose proof requires a little argument. We provide quite a detailed proof that completeness follows from $\omega_{1}$-saturation. Further applications of saturation will follow the same pattern and will not be detailed to the same extent.

Proposition 1. Let $\mathcal{M}$ be an internal $C^{*}$-algebra. Every Cauchy sequence in $\operatorname{Fin}(\mathcal{M})$ converges in $\operatorname{Fin}(\mathcal{M})$.

Proof. Let $\left(x_{n}\right)_{n \in \mathbb{N}}$ be a Cauchy sequence in $\operatorname{Fin}(\mathcal{M})$. For each $k \in \mathbb{N}^{+}$let $n_{k}$ be such that

$$
\forall m, n>n_{k}\left({ }^{\circ}\left\|x_{n}-x_{m}\right\|<\frac{1}{2 k}\right) .
$$

Then $\forall m, n>n_{k}\left(\left\|x_{n}-x_{m}\right\|<\frac{1}{k}\right)$. Without loss of generality, assume that $h<k \Rightarrow n_{h}<n_{k}$. Let $M \in \mathbb{N}$ be such that $\left\|x_{n}\right\|<M$ for all $n \in \mathbb{N}$.

Let $S$ be the internal set of all internal sequences from ${ }^{*} \mathbb{N}$ to $\mathcal{M}$.

Let $A_{k}, k \in \mathbb{N}^{+}$, be the set of internal sequences $\bar{y}=\left(y_{n}\right)_{n \in * \mathbb{N}}$ that satisfy the following properties:

1. $\bar{y} \in S$;

2. $\forall n \in{ }^{*} \mathbb{N}\left(\left\|y_{n}\right\|<M\right)$;

3. $\forall m \leq n_{k}\left(y_{n}=x_{n}\right)$;

4. $\forall m, n \in{ }^{*} \mathbb{N}\left(m, n>n_{k} \Rightarrow\left\|y_{m}-y_{n}\right\|<1 / k\right)$.

For each $k \in \mathbb{N}^{+}$, the set $A_{k}$ is internal by the Internal Definition Principle (see the Introduction). Furthermore, the family $\left\{A_{k}: k \in \mathbb{N}^{+}\right\}$has the finite intersection property: let $k \in \mathbb{N}^{+}$and let $\bar{y}$ be the sequence defined as follows:

$$
y_{n}= \begin{cases}x_{n} & \text { if } n \leq n_{k} \\ x_{n_{k}} & \text { if } n>n_{k}\end{cases}
$$

Then $\bar{y} \in A_{1} \cap \cdots \cap A_{k}$. By Saturation, let $\bar{y} \in \bigcap_{k \in \mathbb{N}^{+}} A_{k}$. Fix any $N \in{ }^{*} \mathbb{N} \backslash \mathbb{N}$. Then $y_{N} \in \operatorname{Fin}(\mathcal{M})$ and, for all $k \in \mathbb{N}^{+}$,

$$
\forall n \in \mathbb{N}\left(n>n_{k} \rightarrow\left\|x_{n}-y_{N}\right\|<1 / k\right)
$$

Therefore ${ }^{\circ}\left\|x_{n}-y_{N}\right\| \rightarrow 0$ as $\mathbb{N} \ni n \rightarrow \infty$.

Now we continue the construction of the nonstandard hull. Let $\mathcal{M}$ be as above and let $I=\{x \in \mathcal{M}:\|x\| \approx 0\}$. Notice that $x \in I \Leftrightarrow{ }^{\circ}\|x\|=0$. It is easy to check that $I$ is a closed ideal in $\operatorname{Fin}(\mathcal{M})$. Let $\widehat{\mathcal{M}}=\operatorname{Fin}(\mathcal{M}) / I$ be the quotient algebra. 
We let $\hat{x}=x+I$. By basic results on quotients of ordinary semi-normed algebras, $\widehat{\mathcal{M}}$ is an ordinary Banach self-adjoint algebra with respect to the operations canonically defined on the quotient and with respect to the norm

$$
\|\hat{x}\|=\inf _{y \in I}^{\circ}\|x+y\|={ }^{\circ}\|x\| .
$$

Notice that the context prevents any ambiguity in the use of symbol \| $\|$.

Clearly, the norm on $\widehat{\mathcal{M}}$ satisfies the $C^{*}$-condition $\left\|(\hat{x})^{*} \hat{x}\right\|=(\|\hat{x}\|)^{2}$. Therefore $\widehat{\mathcal{M}}$ is a $C^{*}$-algebra, which is called the nonstandard hull of $\mathcal{M}$.

If $* \mathcal{M}$ is a nonstandard extension of an ordinary $\mathcal{X}$ algebra $\mathcal{M}$, we still write $\widehat{\mathcal{M}}$ for $\widehat{* \mathcal{M}}$. As previously noticed, the context prevents any ambiguity. Moreover ${ }^{*} \mathcal{M}$ is uniquely determined by $\mathcal{M}$, once a nonstandard universe has been fixed.

In the literature one can find an extensive study of the properties of the nonstandard hull $\widehat{\mathcal{M}}$ of an ordinary $\mathcal{X}$ algebra $\mathcal{M}$. Here we just mention that the norm on $\widehat{\mathcal{M}}$ is always complete (even when $\mathcal{M}$ is semi-normed); $\mathcal{M}$ isometrically embeds into $\widehat{\mathcal{M}}$; if $\mathcal{M}$ is infinite dimensional then $\widehat{\mathcal{M}}$ is nonseparable. Moreover it holds that, for every finite dimensional subalgebra $\mathcal{N}$ of $\widehat{\mathcal{M}}$ and every positive real $\epsilon$, there exists an $\epsilon$-isometry from $\mathcal{N}$ into $\mathcal{M}$. Roughly speaking, this means that $\mathcal{N}$ can be embedded into $\mathcal{M}$ "up to any prescribed degree of accuracy". This is the property of finite representability of $\widehat{\mathcal{M}}$ into $\mathcal{M}$.

We finish this section with a comment that might be of interest to functional analysts. In order to prove that the quotient of an ordinary $C^{*}$-algebra $\mathcal{M}$ with respect to a closed ideal $I$ is a $C^{*}$-algebra, the key step is to prove that the quotient norm satisfies the $C^{*}$-condition. To do that one uses an approximate unit for $I$ (see [5, II.5]). Notice that proving the $C^{*}$-condition for the norm defined on a nonstandard hull is completely straightforward.

\section{An extension of Luxemburg's construction}

In this section we want to introduce a slightly more general nonstandard hull construction obtained by factoring out the finite elements $\operatorname{Fin}(\mathcal{M})$ of an internal $X$ algebra $\mathcal{M}$ with respect to a closed ideal $J$. Let us assume that $\mathcal{M}$ is an internal $C^{*}$-algebra. Notation and results from Section 3 are in force. Let $\widetilde{\mathcal{M}}=\operatorname{Fin}(\mathcal{M}) / J$ and $\tilde{x}=x+J$. We claim that $\widetilde{\mathcal{M}}$ is a $C^{*}$-algebra with respect to the operations canonically defined on the quotient and with respect to the norm

$$
\|\tilde{x}\|=\inf _{y \in J}^{\circ}\|x+y\|
$$

To prove the above claim, the key observations are that $I \subseteq J$ and $\widehat{J}=J / I$ is a closed ideal in the nonstandard hull $\widehat{\mathcal{M}}$ of $\mathcal{M}$. Therefore $\widehat{\mathcal{M}} / \widehat{J}$ is a $C^{*}$-algebra with respect to the norm

$$
\|\hat{x}+\widehat{J}\|=\inf _{\hat{y} \in \hat{J}}\|\hat{x}+\hat{y}\|=\inf _{y \in J}^{\circ}\|x+y\|
$$


Moreover, the map $\pi: \widehat{\mathcal{M}} \rightarrow \widetilde{\mathcal{M}} ; \hat{x} \mapsto \tilde{x}$ is a surjective homomorphism such that ker $\pi=\widehat{J}$. Hence $\widehat{\mathcal{M}} / \widehat{J}$ is isomorphic to $\widetilde{\mathcal{M}}$ and the claim is proved. It worths recalling that an injective homomorphism of $C^{*}$-algebras is an isometry (see [5, II.2.2.9]).

By arguing as in the proof of [24, Theorem 3.22] (but see also [3], on which the content of sections 3.2 and 3.3 of [24] is based), one can show that the self-adjoint elements $\operatorname{Re}(\mathcal{M})$ of $\mathcal{M}$ satisfy the condition $(\operatorname{Re}(\mathcal{M}) \cap \operatorname{Fin}(\mathcal{M}))^{\sim}=\operatorname{Re}(\widetilde{\mathcal{M}})$, from which we get the following for the positive elements: $\left(\operatorname{Fin}\left(\mathcal{M}^{+}\right)\right)^{\sim}=(\widetilde{\mathcal{M}})^{+}$ Technically speaking, one says that self-adjoint and positive elements are liftable. General liftability results, analogous to those proved in [24] (see [13] for their formulation in ultraproduct language), seem not hold for arbitrary $\mathcal{M}$ and $J$.

We stress that liftability is crucial for sake of proving properties of $\widetilde{\mathcal{M}}$ by working inside the internal algebra $\mathcal{M}$, where all the nonstandard techniques can be exploited.

\subsection{Tracial nonstandard hull}

We recall that a representation of an ordinary $C^{*}$-algebra $\mathcal{M}$ is a homomorphism from $\mathcal{M}$ to $\mathcal{B}(\mathcal{H})$, for some Hilbert space $\mathcal{H}$. A faithful representation is an injective one. It follows from [5, II.2.2.9] that a faithful representation is isometric.

If $\mathcal{M}$ is an ordinary (internal) $C^{*}$-algebra, we denote by $\operatorname{Proj}(\mathcal{M})$ the (internal) set of its (internal) projections. We recall that, if $p, q$ are projections in $\mathcal{B}(\mathcal{H}), p<q$ simply means that $\operatorname{ran}(p) \subset \operatorname{ran}(q)$.

In Section 3, we already mentioned that, with the exception of the (standard) finite dimensional case, the nonstandard hull of an internal $C^{*}$-algebra cannot be faithfully represented as an ordinary von Neumann algebra. We sketch a nonstandard proof of this fact as a corollary of results, here stated without proof, which are instances of more general results from [3] (they also appear in [24]). All of them have corresponding ultraproduct versions.

In the next three results, $\mathcal{M}$ is an internal $C^{*}$-subalgebra of the internal $C^{*}$-algebra of bounded linear operators on some internal Hilbert space $\mathcal{H}$. It worths recalling that the nonstandard hull $\widehat{\mathcal{M}}$ of $\mathcal{M}$ is a $C^{*}$-subalgebra of the ordinary algebra $\mathcal{B}(\widehat{\mathcal{H}})$, where $\widehat{\mathcal{H}}$ is the nonstandard hull of the internal Hilbert space $\mathcal{H}$. It is easy to verify that $\widehat{\mathcal{H}}$ is an ordinary Hilbert space. Therefore $\widehat{\mathcal{M}}$ is an algebra of operators.

Proposition 2. For any $\widehat{a} \in \operatorname{Proj}(\widehat{\mathcal{M}})$ there is $p \in \operatorname{Proj}(\mathcal{M})$ such that $\widehat{a}=\widehat{p}$.

A brief comment on the preceding proposition: it might well be the case that $\widehat{a} \in \operatorname{Proj}(\widehat{\mathcal{M}})$ even if $a \notin \operatorname{Proj}(\mathcal{M})$. In any case, Proposition 2 ensures that there exists $p \in \operatorname{Proj}(\mathcal{M})$ such that $p \approx a$. This $p$ is called a lifting of $\widehat{a}$.

Proposition 3. Let $p_{0}, \ldots, p_{n} \in \operatorname{Proj}(\mathcal{M})$ be such that $\widehat{p}_{0}<\cdots<\widehat{p}_{n}$. Then there exist $q_{0}, \ldots, q_{n} \in \operatorname{Proj}(\mathcal{M})$ such that $q_{0}<\cdots<q_{n}$ and $\widehat{p}_{i}=\widehat{q}_{i}$ for all $0 \leq i \leq n$.

Proposition 4. If the nonstandard hull $\widehat{\mathcal{M}}$ of $\mathcal{M}$ contains is a strictly increasing family $\left\{\widehat{p}_{n}\right\}_{n \in \mathbb{N}}$ of projections, then $\widehat{\mathcal{M}}$ is not a von Neumann algebra. 
Corollary 5. The following are equivalent for an internal $C^{*}$-algebra $\mathcal{M}$ :

1. $\mathcal{M}$ is (standard) finite dimensional;

2. $\widehat{\mathcal{M}}$ can be faithfully represented as a von Neumann algebra.

Proof. (1) $\Rightarrow(2)$ This follows by Transfer of the result that an ordinary finite dimensional $C^{*}$-algebra is isomorphic to a finite direct sum of internal matrix algebras of finite dimension over $\mathbb{C}$. Therefore $\widehat{\mathcal{M}}$ is a finite direct sum of matrix algebras over $\mathbb{C}$, each having standard finite dimension. Hence $\widehat{\mathcal{M}}$ is a von Neumann algebra.

$(2) \Rightarrow(1)$ Without loss of generality, let $\widehat{\mathcal{M}}$ be infinite dimensional and von Neumann. Then, for each $n \in \mathbb{N}$, there exists in $\widehat{\mathcal{M}}$ an increasing family $\widehat{p}_{0}<\cdots<\widehat{p}_{n}$ of projections. (Recall that $\widehat{\mathcal{M}}$ is generated by its own projections.) By Proposition 2 we can assume that $p_{i} \in \operatorname{Proj}(\mathcal{M})$, for all $1 \leq i \leq n$. Hence Proposition 3 applies and, in $\mathcal{M}$, there are finite arbitrarily long strictly increasing sequences of projections. By Saturation there exist $N \in{ }^{*} \mathbb{N} \backslash \mathbb{N}$ and a strictly increasing sequence $\left(p_{K}\right)_{K<N}$ of projections in $\mathcal{M}$. Hence, by Proposition $4, \widehat{\mathcal{M}}$ is not a von Neumann algebra. Therefore, if $\widehat{\mathcal{M}}$ is von Neumann, it is finite dimensional and so is $\mathcal{M}$.

Indeed there is a modified hull construction that, applied to any internal $C^{*}$-algebra which admits an internal tracial state, produces an ordinary $C^{*}$-algebra that has a faithful representation as a von Neumann algebra. In this subsection we outline this construction.

Let $\mathcal{M}$ be an internal, unital $C^{*}$-algebra with norm \|\| . The notation and the results of Sections 3 and 4 are in force.

Let $\tau: \mathcal{M} \rightarrow{ }^{*} \mathrm{C}$ be an internal tracial state, namely a positive linear functional of norm one (equivalently: $\tau(1)=1$ ) with the property that $\tau\left(x^{*} x\right)=\tau\left(x x^{*}\right)$, for all $x \in \mathcal{M}$.

By [5, II.3.1.8], $\|x\| \|=\tau\left(x^{*} x\right)^{1 / 2}$ is a seminorm on $\mathcal{M}$ with the property that $\||x\|\mid \leq\| x \|$, for all $x \in \mathcal{M}$. This will be useful in the following.

Using also [5, II.3.1.9(ii)], we get that

$$
J_{\tau}=\left\{x \in \operatorname{Fin}(\mathcal{M}): \tau\left(x^{*} x\right) \approx 0\right\}
$$

is a closed ideal in $\operatorname{Fin}(\mathcal{M})$ with respect to the seminorm ${ }^{\circ}\|\|$. As shown in Section 4 , the quotient $\widetilde{\mathcal{M}}^{\tau}=\operatorname{Fin}(\mathcal{M}) / J_{\tau}$ inherits the structure of a $C^{*}$-algebra. Recall that $\|\tilde{x}\|=\inf _{y \in J_{\tau}} \circ\|x+y\|$.

By [5, II.3.1.8], we get that $x^{*} y^{*} y x \leq\|y\|^{2} x^{*} x$, for all $x, y \in \operatorname{Fin}(\mathcal{M})$. From $[5$, II.3.1.9] it follows that

$$
J_{\tau}=\left\{x \in \operatorname{Fin}(\mathcal{M}): \tau\left(x^{*} x\right) \approx 0\right\}
$$

is a closed ideal in $\operatorname{Fin}(\mathcal{M})$ with respect to the seminorm ${ }^{\circ}\|\|$. As shown in Section 4 , the quotient $\widetilde{\mathcal{M}}^{\tau}=\operatorname{Fin}(\mathcal{M}) / J_{\tau}$ inherits the structure of a $C^{*}$-algebra. 
With the above notation in force, we can state and prove the following:

Theorem 6. Let $\mathcal{M}$ be an internal $C^{*}$-algebra and let $\tau: \mathcal{M} \rightarrow{ }^{*} \mathbb{C}$ be an internal tracial state. Then $\widetilde{\mathcal{M}}^{\tau}$ has a faithful representation as a von Neumann algebra.

Proof. First of all observe that $\tau$ induces a tracial state $\theta$ on $\widetilde{\mathcal{M}}^{\tau}$ defined by $\theta(\tilde{x})={ }^{\circ} \tau(x)$. Notice in particular that from [5, II.6.2.6] we get that $\tau(x) \approx \tau(y)$ when $x-y \in J_{\tau}$, so $\theta$ is well-defined.

Let us consider the GNS representation of $\widetilde{\mathcal{M}}^{\tau}$ associated to $\theta$. (For its construction, see [5, II.6.4]). We notice that, under our assumptions, there is a simplification with respect to the general construction, due to the fact that

$$
\theta\left(\tilde{x}^{*} \tilde{x}\right)=0 \Leftrightarrow \tilde{x}=0 .
$$

Therefore $\theta$ is faithful and the pre-inner product $\langle\tilde{x}, \tilde{y}\rangle=\theta\left(\tilde{y}^{*} \tilde{x}\right)$ is actually an inner product on $\widetilde{\mathcal{M}}^{\tau}$. A saturation argument similar to that presented in Section 3 shows that $\widetilde{\mathcal{M}}^{\tau}$ is complete with respect to such inner product. We denote by $\|\cdot\|_{2}$ the corresponding norm.

Let $L_{\tilde{x}}: \widetilde{\mathcal{M}}^{\tau} \rightarrow \widetilde{\mathcal{M}}^{\tau}$ be the operator of left multiplication by $\tilde{x}$. Clearly, the homomorphism $\pi: \widetilde{\mathcal{M}}^{\tau} \rightarrow \mathcal{B}\left(\widetilde{\mathcal{M}}^{\tau}\right)$ defined by $\pi(\tilde{x})=L_{\tilde{x}}$ is injective, hence isometric by [5, II.2.2.9]: $\|\tilde{x}\|=\left\|L_{\tilde{x}}\right\|_{\mathcal{B}\left(\widetilde{\mathcal{M}}^{\tau}\right)}$.

So far we have proved that $\pi$ is a faithful representation of $\widetilde{\mathcal{M}}^{\tau}$. Next we prove that that $\pi\left[\widetilde{\mathcal{M}}^{\tau}\right]$ is closed with respect to the SOT (see the Introduction).

Let $\left(L_{\tilde{x}_{i}}\right)_{i \in I} \subset \widetilde{\mathcal{M}}^{\tau}$ be a net converging to some $f \in \mathcal{B}\left(\widetilde{\mathcal{M}}^{\tau}\right)$ with respect to the SOT. Then $\left(\left\|\widetilde{x}_{i}\right\|\right)_{i \in I}$ is bounded and we can pick the $x_{i}{ }^{\prime}$ s so that there exists $r \in \mathbb{R}^{+}$for which $\left\|x_{i}\right\| \leq r$ for all $i \in I$.

Let $\mathcal{M}_{r}=\{x \in \mathcal{M}:\|x\| \leq r\}$ and $d(x, y)=\|x-y\|, x, y \in \mathcal{M}_{r}$, where \|\|$\|$ is the semi-norm associated to the tracial state $\tau$ defined at the beginning of this subsection. Then $\left(\mathcal{M}_{r}, d\right)$ is an internal pseudometric space. Note that the galaxy of 0 , namely the set $\left\{y \in \mathcal{M}_{r}:\|y\|\right.$ is finite $\}$, is just $\mathcal{M}_{r}$. Let $\bar{x}=\left\{y \in \mathcal{M}_{r}\right.$ : $d(x, y) \approx 0\}$ and $\overline{\mathcal{M}}_{r}=\left\{\bar{x}: x \in \mathcal{M}_{r}\right\}$.

Define a distance $\bar{d}$ on $\overline{\mathcal{M}}_{r}$ as follows: $\bar{d}(\bar{x}, \bar{y})={ }^{\circ} d(x, y)$. By Saturation, $\left(\overline{\mathcal{M}}_{r}, \bar{d}\right)$ is a complete metric space. Moreover, $\bar{d}(\bar{x}, \bar{y})=\|\tilde{x}-\tilde{y}\|_{2}$, for all $x, y \in \mathcal{M}_{r}$.

By SOT-convergence, $\left(\widetilde{x}_{i}\right)_{i \in I}=\left(L_{\widetilde{x}_{i}}(\tilde{1})\right)_{i \in I}$ converges to $f(\tilde{1})$ with respect to \|\|$_{2}$, hence it is a Cauchy net with respect to the latter norm. Thus $\left(\bar{x}_{i}\right)_{i \in I}$ is Cauchy in $\left(\overline{\mathcal{M}}_{r}, \bar{d}\right)$. By completeness of $\left(\overline{\mathcal{M}}_{r}, \bar{d}\right)$ there exists $x \in \mathcal{M}_{r}$ such that $\bar{d}\left(\overline{x_{i}}-\bar{x}\right) \rightarrow 0$. Hence $\left\|\widetilde{x}_{i}-\widetilde{x}\right\|_{2} \rightarrow 0$ and so $L_{\widetilde{x}}=f$. This proves that $\widetilde{\mathcal{M}}^{\tau}$ is closed with respect to the SOT.

Versions of the previous theorem stated in ultraproduct language or proved in specific cases already appear in the literature, see for instance [21], [17], [16] or [14]. To the best of our knowledge, the above proof is novel. 


\subsection{Weak nonstandard hull}

In this subsection, we first present the weak nonstandard hull construction (see [24]) in a way that fits into the setting of Section 4 . Then we comment on a slight generalization of such construction.

Let ${ }^{*} \mathcal{M}$ be the nonstandard extension of an ordinary Banach space $\mathcal{M}$. Let $\mathcal{M}^{\prime}$ be the dual of $\mathcal{M}$. For notational simplicity we write $\phi$ also for the nonstandard extension ${ }^{*} \phi$ of $\phi \in \mathcal{M}^{\prime}$. Let $\mathcal{M}_{1}^{\prime}$ be the closed unit ball of $\mathcal{M}^{\prime}$.

Let $J_{\mathrm{w}}=\operatorname{Fin}\left({ }^{*} \mathcal{M}\right) \cap\left\{x \in{ }^{*} \mathcal{M}: \phi(x) \approx 0\right.$ for all $\left.\phi \in \mathcal{M}_{1}^{\prime}\right\}$. Clearly $J_{\mathrm{w}}$ contains the subspace $\left\{x \in \operatorname{Fin}\left({ }^{*} \mathcal{M}\right):\|x\| \approx 0\right\}$ of the ordinary semi-normed space $\operatorname{Fin}\left({ }^{*} \mathcal{M}\right)$. Moreover, it is easy to check that $J_{\mathrm{w}}$ is a closed subspace of $\operatorname{Fin}\left({ }^{*} \mathcal{M}\right)$. Then, by the remark made at the beginning of this section, we get at once that $\widetilde{\mathcal{M}}^{\mathrm{w}}=\operatorname{Fin}\left({ }^{*} \mathcal{M}\right) / J_{\mathrm{w}}$ is a Banach space, called the weak nonstandard hull of $\mathcal{M}$.

In [24], the author proves that $\widetilde{\mathcal{M}}^{\mathrm{w}}$ is isometrically isomorphic to the bidual $\mathcal{M}^{\prime \prime}$ of $\mathcal{M}$.

Actually, $\widetilde{\mathcal{M}}^{\mathrm{w}}$ is first presented in [24] as a nonstandard hull obtained from a family of internal seminorms (those canonically associated to the elements of $\left.\mathcal{M}_{1}^{\prime}\right)$. If $\mathcal{M}$ is an internal Banach space and $F \subseteq \mathcal{M}_{1}^{\prime}$, then the above construction can be repeated by letting

$$
J_{F}=\operatorname{Fin}(\mathcal{M}) \cap\{x \in \mathcal{M}: \phi(x) \approx 0 \text { for all } \phi \in F\}
$$

and $\widetilde{\mathcal{M}}^{F}=\operatorname{Fin}(\mathcal{M}) / J_{F}$. By the remark made at the beginning of this section, $\widetilde{\mathcal{M}}^{F}$ is a Banach space. Note that the latter construction includes, as a special case, the original weak nonstandard hull.

\section{Preservation of properties}

In this section we work with $C^{*}$-algebras. We provide a number of results showing that significant properties are preserved and reflected by the nonstandard hull construction. We stress that the same results can be obtained also in the ultraproduct or in the continuous logic setting (see [4]).

We begin with some lifting results in the vein of those in [3]. Most of them involve the notion of projection, because of its crucial role in a $C^{*}$-algebra, if any exists besides 0 and 1 . Actually there are easy examples of so called projectionless $C^{*}$-algebras like $\mathbb{C}$ or $\mathcal{C}([0,1])$. The first example of an infinite dimensional, simple (i.e without proper ideals), projectionless $C^{*}$-algebra is due to Blackadar.

Let $\mathcal{M}$ be a $C^{*}$-algebra. We recall that $u \in \mathcal{M}$ is a partial isometry if $u^{*} u$ is a projection (see [5, II.1.5.7]). Recall also that two projections $p, q \in \mathcal{M}$ are Murrayvon Neumann equivalent in $\mathcal{M}$ (notation: $p \sim q$ ) if there is a partial isometry $u$ such that $u^{*} u=p$ and $u u^{*}=q$ (see [5, II.3.3.3]).

The notation $p \sim q$ should not be confused with $p \approx q$, the latter meaning that $\|p-q\|$ is infinitesimal.

We denote by $U(\mathcal{M})$ the multiplicative group of unitary elements in an (ordinary or internal) $C^{*}$-algebra $\mathcal{M}$. 
Let $\widehat{\mathcal{M}}$ be the nonstandard hull of an internal $C^{*}$-algebra $\mathcal{M}$. If $X \subseteq \mathcal{M}$, we let $\widehat{X}=\{\widehat{x}: x \in \operatorname{Fin}(X)\}$.

A unitary element $u$ in a $C^{*}$-algebra is one such that $u^{*} u=1=u u^{*}$. The following proposition shows that each unitary element of $\widehat{\mathcal{M}}$ is liftable.

Proposition 7. Let $\mathcal{M}$ be an internal $C^{*}$-algebra. Then $U(\widehat{\mathcal{M}})=\widehat{U(\mathcal{M})}$.

Proof. For the nontrivial inclusion, let $\widehat{u} \in U(\widehat{\mathcal{M}})$. A fortiori, $\widehat{u}$ is a partial isometry. Hence, by [24, Theorem 3.22], there exists a partial isometry $v \in \mathcal{M}$ such that $v \approx u$. Therefore $v v^{*}=p \approx 1$ and $v^{*} v=q \approx 1$, for some internal projections $p, q$ on $\mathcal{M}$. By Transfer of [5, II.3.3.4], the condition $p \approx 1$ implies $p \sim 1$. Hence $p=1$. Similarly for $q$. Therefore $v$ is unitary and $\widehat{v}=\widehat{u}$.

Let $\operatorname{Aut}(\mathcal{M})$ be the automorphism group of an ordinary or internal $C^{*}$-algebra $\mathcal{M}$. By an automorphism here we mean what is often referred to as a $*$-automorphism. An inner automorphism $\theta$ is one such that there exists $u \in U(\mathcal{M})$ for which $\theta(x)=u x u^{*}$ for all $x \in \mathcal{M}$. We shall write $\theta_{u}$ to denote the inner automorphism associated to $u \in U(\mathcal{M})$. We denote by $\operatorname{Inn}(\mathcal{M})$ the inner automorphism group.

Let $\widehat{\mathcal{M}}$ be the nonstandard hull of an internal $C^{*}$-algebra $\mathcal{M}$. If $\theta \in \operatorname{Aut}(\mathcal{M})$ then the map $\widehat{\theta}: \widehat{\mathcal{M}} \rightarrow \widehat{\mathcal{M}}$ defined by $\widehat{\theta}(\widehat{x})=\widehat{\theta(x)}$ is in $\operatorname{Aut}(\widehat{\mathcal{M}})$. If $A \subseteq \operatorname{Aut}(\mathcal{M})$, we let $\widehat{A}=\{\widehat{\theta}: \theta \in A\}$.

The next corollary is a straightforward consequence of Proposition 7.

Corollary 8. Let $\mathcal{M}$ be an internal $C^{*}$-algebra. Then $\left.\widehat{\operatorname{Inn}(\mathcal{M}}\right)=\operatorname{Inn}(\widehat{\mathcal{M}})$.

Proof. Since $\widehat{\theta_{u}}=\theta_{\widehat{u}}$, it remains to prove that $\left.\operatorname{Inn}(\widehat{\mathcal{M}}) \subseteq \widehat{\operatorname{Inn}(\mathcal{M}}\right)$. The latter follows from Proposition 7.

We have just shown that all inner automorphisms of $\widehat{\mathcal{M}}$ are liftable to inner automorphisms of $\mathcal{M}$. In the framework of von Neumann algebras, a much more complicated liftability result has been proved for inner automorphisms of tracial ultrapowers of a $\mathrm{II}_{1}$ factor (see [26]).

We recall that two projections $p, q$ in a $C^{*}$-algebra $\mathcal{M}$ are unitarily equivalent in $\mathcal{M}$ if there exists $u \in U(\mathcal{M})$ such that $u p u^{*}=q$ (notation: $p \sim_{u} q$ ).

Corollary 9. Let $\mathcal{M}$ be an internal $C^{*}$-algebra and let $\hat{p}, \hat{q}$ be unitarily equivalent projections in $\widehat{\mathcal{M}}$. Then there exists projections $p^{\prime}, q^{\prime} \in \mathcal{M}$ such that $p^{\prime} \approx p, q^{\prime} \approx q$ and $p^{\prime} \sim_{u} q^{\prime}$

Proof. Let $\widehat{u} \in U(\widehat{\mathcal{M}})$ be such that $\widehat{u} \widehat{p}(\widehat{u})^{*}=\widehat{q}$. By [24, Theorem 3.22] we can assume that $p, q \in \operatorname{Proj}(\mathcal{M})$. By Proposition 7, we can further assume that $u \in U(\mathcal{M})$. Then $u p u^{*} \approx q$ and $u p u^{*} \in \operatorname{Proj}(\mathcal{M})$. 
The previous corollary is analogous to the liftability result for Murray-von Neumann equivalence of projections proved in [24, Lemma 3.7].

A result similar to Corollary 9 holds with respect to the notion of homotopic equivalence. Two projections $p, q$ in a $C^{*}$-algebra $\mathcal{M}$ are homotopic in $\mathcal{M}$ if there exists a continuous mapping $\theta:[0,1] \rightarrow \operatorname{Proj}(\mathcal{M})$ such that $\theta(0)=p$ and $\theta(1)=q$ (notation: $p \sim_{h} q$ ).

Let $\mathcal{M}$ be an internal $C^{*}$-algebra. According to the nonstandard terminology, we say that a mapping $\theta:{ }^{*}[0,1] \rightarrow \mathcal{M}$ is $S$-continuous if

$$
x \approx y \Rightarrow \theta(x) \approx \theta(y) \text { for all } x, y \in \in^{*}[0,1] .
$$

We give the following:

Definition 10. Let $\mathcal{M}$ be an internal $C^{*}$-algebra. Two projections $p, q \in \operatorname{Proj}(\mathcal{M})$ are S-homotopic (notation: $p \sim_{S h} q$ ) if there exists an internal $S$-continuous mapping $\theta:{ }^{*}[0,1] \rightarrow \mathcal{M}$ with the following properties:

1. $\theta(0)=p$;

2. $\theta(1)=q$;

3. $\theta(x) \in \operatorname{Proj}(\mathcal{M})$, for all $\left.x \in *^{*}\right] 0,1[$.

Proposition 11. The following are equivalent for $p, q \in \operatorname{Proj}(\mathcal{M})$, where $\mathcal{M}$ is an internal $C^{*}$-algebra:

1. $p \sim_{S h} q$

2. $\widehat{p} \sim_{h} \widehat{q}$.

Proof. $(\Rightarrow)$ This is a routine nonstandard argument. Let $\theta$ be an internal mapping witnessing $p \sim_{S h} q$. For each $0<\epsilon \in \mathbb{R}$, let

$$
A_{\epsilon}=\left\{\delta \in{ }^{*} \mathbb{R}: \forall x, y \in{ }^{*}[0,1](|x-y|<\delta \rightarrow\|\theta(x)-\theta(y)\|<\epsilon\} .\right.
$$

By the Internal Definition Principle, the set $A_{\epsilon}$ is internal. Since it contains the external set of positive infinitesimals, there exists some positive noninfinitesimal $\delta \in A_{\epsilon}$. Therefore the mapping

$$
\begin{aligned}
\widehat{\theta}:[0,1] & \rightarrow \operatorname{Proj}(\widehat{\mathcal{M}}) \\
x & \mapsto \widehat{\theta(x)}
\end{aligned}
$$

is continuous. Furthermore $\widehat{\theta}$ witnesses $\widehat{p} \sim_{h} \widehat{q}$.

$(\Leftarrow)$ Let $\eta:[0,1] \rightarrow \operatorname{Proj}(\widehat{\mathcal{M}})$ be a continuous function such that $\eta(0)=\widehat{p}$ and $\eta(1)=\widehat{q}$. We work within the framework of the Neometric spaces of Fajardo and Keisler (this is another way of dealing with nonstandard techniques). More precisely, we work in the Huge Neometric Family (see [11]). By [12, Proposition 4.12], the mapping $\eta$ is neocontinuous. It follows from the fact that every compact set its neocompact and from [11, Theorem 4.17] 
that $\eta$ is (uniformly) liftable, namely there exists an internal function $\theta: *[0,1] \rightarrow \mathcal{M}$ such that $\widehat{\theta(x)}=\eta\left({ }^{\circ} x\right)$, for all $x \in *^{*}[0,1]$.

Hence $\theta$ is $S$-continuous; $\theta(0) \approx p ; \theta(1) \approx q$ and $\left.\forall x \in{ }^{*}\right] 0,1[\exists r \in \operatorname{Proj}(\mathcal{M})$ $(\theta(x) \approx r)$. Let

$$
B=\left\{N \in{ }^{*} \mathbb{N}: \forall x \in{ }^{*}[0,1] \exists r \in \operatorname{Proj}(\mathcal{M})(\|\theta(x)-r\|<1 / N)\right\} .
$$

The set $B$ is internal and contains the external set $\mathbb{N}$. Let $N \in\left({ }^{*} \mathbb{N} \backslash \mathbb{N}\right) \cap B$. For $x \in{ }^{*}[0,1]$, let $A_{x}$ be the nonempty set $\{r \in \operatorname{Proj}(\mathcal{M}):\|\theta(x)-r\|<$ $1 / N)\}$.

Let $c h$ be an internal choice function on $\left\{A_{x}: x \in{ }^{*}[0,1]\right\}$ (notice that the latter is internal by the Internal Definition Principle) and let $\xi:{ }^{*}[0,1] \rightarrow$ $\operatorname{Proj}(\mathcal{M})$ be defined by $\xi(0)=p, \xi(1)=q$ and $\xi(x)=\operatorname{ch}\left(A_{x}\right)$, for all $\left.x \in{ }^{*}\right] 0,1\left[\right.$. Such $\xi$ witnesses $p \sim_{S h} q$.

Notice that the argument used in the previous proof shows that Sh-homotopy of projections $p$ and $q$ is equivalent to the apparently weaker condition of existence of an internal $S$-continuous mapping $\theta: *[0,1] \rightarrow \operatorname{Proj}(\mathcal{M})$ with the properties that $\theta(0) \approx p ; \theta(1) \approx q$ and $\left.\forall x \in{ }^{*}\right] 0,1[\exists r \in \operatorname{Proj}(\mathcal{M})(\theta(x) \approx r)$.

If $p, q$ are projections in an internal $C^{*}$-algebra $\mathcal{M}$, then $p \sim_{S h} q$ implies $p \sim_{u}$ $q$. To see that, let $\theta$ be as in the definition of Sh-homotopy and let $N \in{ }^{*} \mathbb{N} \backslash \mathbb{N}$. Then $\theta(i / N) \approx \theta\left(\frac{i+1}{N}\right)$ and hence, by [5, Proposition II.3.3.4], $\theta\left(\frac{i}{N}\right) \sim_{u} \theta\left(\frac{i+1}{N}\right)$, for all $0 \leq i<N$. The conclusion follows by transitivity of $\sim_{u}$.

Notice also that for $p, q$ as above, if $p \sim_{u} q$ then $\operatorname{diag}(p, 0) \sim_{S h} \operatorname{diag}(q, 0)$ in the $C^{*}$-algebra $M_{2}(\mathcal{M})$ of $2 \times 2$ matrices with entries in $\mathcal{M}$. (The proof is the same as [5, Proposition V.1.1.3], completed by the trivial observation that sine and cosine functions are $S$-continuous.)

Therefore $\sim_{S h}$ appears to be, in the internal setting, a natural counterpart of $\sim_{h}$ in the ordinary setting. Of course, $\sim_{h}$ makes perfect sense for internal $C^{*}$-algebras as well, but, as far as its liftability is concerned, Proposition 11 tells us that $\sim_{S h}$ is indeed the right notion.

In the following we investigate whether other properties are preserved and/or reflected by the nonstandard hull construction.

We recall that a $C^{*}$-algebra $\mathcal{M}$ is a factor if its center $Z(\mathcal{M})$ is trivial.

Remark 12. Let $\mathcal{M}$ be an internal $C^{*}$-algebra. It is easy to check that if $\widehat{\mathcal{M}}$ is a factor, then $\mathcal{M}$ itself is a factor. For, suppose not. Since the center of a $C^{*}$-algebra can be faithfully represented as a von Neumann algebra and since every von Neumann algebra is generated by its own projections, there is a nontrivial projection $p \in Z(\mathcal{M})$. Moreover $0 \not \approx p \not 1$, otherwise $p=0$ or $p=1$ (see the argument used in the proof of Proposition 7). Therefore $\widehat{p} \in Z(\widehat{\mathcal{M}})$ is a nontrivial projection.

It is an open problem whether the nonstandard hull of a factor is itself a factor. Even if the answer is likely to be false in general, in the case of the internal von Neumann algebra $\mathcal{M}=\mathcal{B}(\mathcal{H})$ of bounded linear operators on some internal 
Hilbert space $\mathcal{H}$, we have that $\widehat{\mathcal{M}}$ is a factor. To see that, for sake of contradiction let $\hat{p} \in Z(\mathcal{M})$ be a nontrivial projection. (Again we use the fact that $Z(\mathcal{M})$, being a von Neumann algebra, is generated by projections.) By [24, Theorem 3.22], we can assume that $p \in \mathcal{M}$ is a projection, actually a nontrivial one. Let $u \in \operatorname{ker}(p)$ and $v \in \operatorname{ran}(p)$ be unit vectors. Define $f \in \mathcal{M}$ as follows: $f(u)=v$ and $f_{\mid\langle u\rangle}=0$. Then $\|p f-f p\| \geq 1$, contradicting to $\widehat{p} \widehat{f}=\widehat{f} \hat{p}$.

Compare the previous observation with the result, proved in [16], that the tracial nonstandard hull of $M_{N}\left({ }^{*} \mathrm{C}\right)$ with respect to the normalized matrix trace is a factor (indeed a type $\mathrm{II}_{1}$ factor).

Actually, the previous consideration can be strengthened. In order to do that we first recall the a $C^{*}$-algebra $\mathcal{M}$ is weakly central if any two maximal ideals $I_{1}$ and $I_{2}$ of $\mathcal{M}$ coincide exactly when $I_{1} \cap Z(\mathcal{M})=I_{2} \cap Z(\mathcal{M})$. The class of weakly central $C^{*}$-algebras contains that of von Neumann algebras. (See [23].)

An element $v$ of a $C^{*}$-algebra $\mathcal{M}$ induces an inner derivation $D(v, \mathcal{M})$, given by $D(v, \mathcal{M})(w)=v w-w v$, for $w \in \mathcal{M}$. Clearly, $D(v, \mathcal{M})$ is a bounded linear operator. Let

$$
\|D(v, \mathcal{M})\|=\sup \{\|v w-w v\|:\|w\| \leq 1 \text { and } w \in \mathcal{M}\}
$$

be the operator norm and let $\mathrm{d}(v, Z(\mathcal{M}))$ be the distance between $v$ and $Z(\mathcal{M})$. As remarked at the end of [27], the results proved therein immediately imply the following:

Proposition 13. Let $\mathcal{M}$ be a weakly central $C^{*}$-algebra. Then, for all $v \in M$,

$$
\mathrm{d}(v, Z(\mathcal{M})) \leq\|D(v, \mathcal{M})\| .
$$

We apply the previous result to prove the following:

Theorem 14. Le $\mathcal{M}$ be an internal weakly central $C^{*}$-algebra. Then

$$
Z(\widehat{\mathcal{M}})=\widehat{Z(\mathcal{M})}
$$

Proof. For the nontrivial inclusion, let $\widehat{v} \in Z(\widehat{\mathcal{M}})$. Then $v w \approx w v$, for all $w \in \operatorname{Fin}(\mathcal{M})$ and so $\|D(v, \mathcal{M})\|$, being a supremum of infinitesimals over an internal set, is itself infinitesimal. From Proposition 13, we get that $\mathrm{d}(v, Z(\mathcal{M})) \approx 0$. Let $w \in Z(\mathcal{M})$ be such that $\|v-w\| \approx 0$. Then $\|w\|$ is finite and $\widehat{v}=\widehat{w} \in$ $\widehat{Z(\mathcal{M})}$.

Corollary 15. Let $\mathcal{M}$ be an internal weakly central $C^{*}$-algebra. Then

$$
\mathcal{M} \text { is a factor } \Leftrightarrow \widehat{\mathcal{M}} \text { is a factor. }
$$

In particular the above holds for an internal von Neumann algebra $\mathcal{M}$.

Proof. $(\Rightarrow)$ Straightforward from Theorem 14 .

$(\Leftarrow)$ See Remark 12 . 
Recalling that nonstandard hulls generalize ultraproducts, we get from Corollary 15 that the ultraproduct of factor von Neumann algebras is a factor $C^{*}$-algebra (but, in general, not a von Neumann algebra, as pointed out at the beginning of Section 3).

An analogue of Theorem 14 can be proved for the class of primitive $C^{*}$-algebras. A $C^{*}$-algebra is primitive if it has a faithful irreducible representation. The notion of faithful representation has been introduced in Section 4.1. A representation is irreducible if it has no nontrivial closed invariant subspaces (see [5, II.6.1]). Primitive $C^{*}$-algebras, and more generally prime $C^{*}$-algebras (of which the primitive ones form a proper subclass, see below), are regarded, within the class of $C^{*}$-algebras, as analogues of factors among von Neumann algebras (so, a sort of "building blocks"). Thus it is not surprising that a result along the line of Theorem 14 can be proved for the class of primitive $C^{*}$-algebras. We use the following (see [27, Theorem 2.2.]):

Proposition 16. Let $\mathcal{M}$ be a primitive $C^{*}$-algebra. Then, for all $v \in \mathcal{M}$, there exist a unique scalar $\lambda(v)$ such that

$$
\|D(v, \mathcal{M})\|=2\|v-\lambda(v) 1\|
$$

Notice that from the previous proposition we get at once the well known fact that every primitive $C^{*}$-algebra is a factor. Indeed, if $\mathcal{M}$ is an internal primitive $C^{*}$-algebra, then $\widehat{\mathcal{M}}$ is a factor. For, let $\widehat{v} \in Z(\widehat{\mathcal{M}})$. Then, by the same argument used in the proof of Theorem 14 , we get $\|D(v, \mathcal{M})\| \approx 0$. Let $\lambda(v)$ be as in Proposition 16. Therefore $\lambda(v) \in \operatorname{Fin}\left({ }^{*} \mathbb{C}\right)$ and $\widehat{v}=\widehat{\lambda(v)} \in \mathbb{C} 1$. This result will be strengthened by Corollary 22.

In the following we continue our investigation on which properties of $C^{*}$-algebras and their representations are preserved by the nonstandard hull construction. It is convenient to prove first a number of more general results concerning homomorphisms.

Let $\mathcal{M}, \mathcal{N}$ be internal $C^{*}$-algebras and let $f: \mathcal{M} \rightarrow \mathcal{N}$ be an internal homomorphism. Since homomorphisms of $C^{*}$-algebras are nonexpansive [5, Corollary II.1.6.6], the map $\widehat{f}: \widehat{\mathcal{M}} \rightarrow \widehat{\mathcal{N}}, \widehat{x} \mapsto \widehat{f(x)}$ is well-defined. It is straightforward to check that $\widehat{f}$ is actually a homomorphism. We recall the following:

Fact 17. Let $f: \mathcal{M} \rightarrow \mathcal{N}$ be a homomorphism of ordinary $C^{*}$-algebras. Then ker $f$ is a closed ideal in $\mathcal{M}$ and the quotient $\mathcal{M} /(\operatorname{ker} f)$ is isometrically isomorphic to the $C^{*}$-algebra $f[\mathcal{M}]$. (See [5, Corollary II.5.1.2]). Therefore, for all $0<\epsilon$ there exists $y \in \operatorname{ker} f$ such that $\|x+y\|<\|f(x)\|+\epsilon$.

The next proposition is a direct consequence of Fact 17.

Proposition 18. Let $\mathcal{M}, \mathcal{N}$ be internal $C^{*}$-algebras and let $f: \mathcal{M} \rightarrow \mathcal{N}$ be an internal homomorphism. Then

$$
\widehat{\operatorname{ker} f}=\operatorname{ker} \widehat{f} .
$$


Proof. The left-to-right inclusion is trivial. As for the converse, let $\widehat{x} \in \operatorname{ker} \widehat{f}$. Then $\|f(x)\| \approx 0$. By Transfer of Fact 17, there exists $y \in \operatorname{ker} f$ such that $\|x-y\| \approx 0$. Hence $\widehat{x}=\widehat{y} \in \widehat{\operatorname{ker} f}$.

Proposition 19. Let $J$ be an internal closed ideal in an internal $C^{*}$-algebra $\mathcal{M}$. Then the map $\widehat{q}: \widehat{\mathcal{M}} / \widehat{J} \rightarrow \widehat{\mathcal{M} / J}, \widehat{x}+\widehat{J} \mapsto \widehat{x+J}$ is an isometric isomorphism.

Proof. By Transfer of the results mentioned in Fact $17, \mathcal{M} / J$ is an internal $C^{*}$-algebra and $q: \mathcal{M} \rightarrow \mathcal{M} / J, x \mapsto x+J$, is an internal homomorphism. Then Proposition 18 and Fact 17 yield the conclusion.

We have already mentioned that we can form the nonstandard hull $\widehat{\mathcal{H}}$ of an internal Hilbert space $\mathcal{H}$ in the same way as with an internal normed space. Moreover $\widehat{\mathcal{H}}$ is itself a Hilbert space with respect to the standard part of the inner product of $\mathcal{H}$.

Let $\pi: \mathcal{M} \rightarrow \mathcal{B}(\mathcal{H})$ be an internal representation of a $C^{*}$-algebra $\mathcal{M}$. We can regard $\widehat{\mathcal{B}(\mathcal{H})}$ as a $C^{*}$-subalgebra of $\mathcal{B}(\widehat{\mathcal{H}})$, by identifying $\widehat{f} \in \widehat{\mathcal{B}(\mathcal{H})}$ with $F: \widehat{\mathcal{H}} \rightarrow \widehat{\mathcal{H}}$ defined by $\widehat{u} \mapsto \widehat{f(u)}$. (See [24, §3.3.1].)

Therefore $\pi$ induces a representation $\widehat{\pi}$ of $\widehat{\mathcal{M}}$ on $\widehat{\mathcal{H}}$.

Theorem 20. Let $\pi: \mathcal{M} \rightarrow \mathcal{B}(\mathcal{H})$ be an internal representation of a $C^{*}$-algebra $\mathcal{M}$. Then

$$
\pi \text { is irreducible } \Leftrightarrow \hat{\pi} \text { is irreducible. }
$$

Proof. $(\Rightarrow)$ Since $\pi$ is irreducible, it follows from a generalization of Schur's Lemma (see [25, Theorem 4.4.12]) that the double commutant $\pi[\mathcal{M}]^{\prime \prime}$ of $\pi[\mathcal{M}]$ is $\mathcal{B}(\mathcal{H})$. Let $u, v \in \mathcal{H}$ be unit vectors and let $T \in \mathcal{B}(\mathcal{H})$ be defined by $T(u)=v$ and $T(w)=0$, when $w \in\langle u\rangle^{\perp}$. Fix $0 \approx \epsilon>0$. An application of Kaplansky Density Theorem $[7,44.1(\mathrm{a})]$ yields $x \in \mathcal{M}$ satisfying

$$
\|\pi(x)\| \leq 1 \text { and }\|\pi(x)(u)-v\|<\epsilon .
$$

By Transfer of Fact 17, there exists $y \in \mathcal{M}$ such that $\pi(x)=\pi(y)$ and $\|y\|<\|\pi(x)\|+1$. Without loss of generality we can therefore assume $x \in \operatorname{Fin}(\mathcal{M})$. Finally, from $(2)$, we get $\widehat{\pi}(\widehat{x})(\widehat{u})=\widehat{v}$.

Being $u, v$ arbitrary unit vectors, we conclude that $\widehat{\pi}$ is irreducible.

$(\Leftarrow)$ By contraposition. Let $\mathcal{H}_{1}$ be an internal nontrivial Hilbert subspace of $\mathcal{H}$ such that $\pi(x)\left[\mathcal{H}_{1}\right] \subseteq \mathcal{H}_{1}$, for all $x \in \mathcal{M}$.

Then $\widehat{\mathcal{H}_{1}}$ is a nontrivial Hilbert subspace of $\widehat{\mathcal{H}}$ and $\widehat{\pi}(\widehat{x})\left[\widehat{\mathcal{H}_{1}}\right] \subseteq \widehat{\mathcal{H}_{1}}$, for all $x \in \operatorname{Fin}(\mathcal{M})$.

The following holds:

Corollary 21. Let $\pi: \mathcal{M} \rightarrow \mathcal{B}(\mathcal{H})$ be an internal representation of a $C^{*}$-algebra $\mathcal{M}$. Then

$$
\pi \text { is faithful } \Leftrightarrow \widehat{\pi} \text { is faithful. }
$$


Proof. The left-to-right implication follows immediately from Proposition 18. The converse implication is straightforward, by contraposition.

Corollary 22. Let $\mathcal{M}$ be an internal $C^{*}$-algebra. Then

$$
\mathcal{M} \text { is primitive } \Leftrightarrow \widehat{\mathcal{M}} \text { is primitive. }
$$

Analogues of Theorem 20 and Corollary 22 have been proved in [13] for ultraproducts of $C^{*}$-algebras. In our opinion, the use of nonstandard techniques leads to simple, intuitive proofs.

A $C^{*}$-algebra $\mathcal{M}$ is prime if whenever $J$ and $J$ are ideals of $\mathcal{M}$ with $I \cap J=\{0\}$, either $I$ or $J$ is $\{0\}$. In order to formulate conditions equivalent to primeness that will be used in the sequel, we define $m_{u v}: \mathcal{M} \rightarrow \mathcal{M}$ as follows: $m_{u v}(w)=u w v$, when $u, v \in \mathcal{M}$.

Proposition 23. The following are equivalent for a $C^{*}$-algebra $\mathcal{M}$ :

1. $\mathcal{M}$ is prime;

2. whenever $u, v \in \mathcal{M}$ are nonzero elements, then there exists $w \in \mathcal{M}$ such that $u w v \neq 0$;

3. $\left\|m_{u v}\right\|=\|u\|\|v\|$, for all $u, v \in \mathcal{M}$.

Proof. See [5, II.5.4.5] and [20] or [8].

Proposition 24. Let $\mathcal{M}$ be an internal $C^{*}$-algebra. Then

$$
\mathcal{M} \text { is prime } \Leftrightarrow \widehat{\mathcal{M}} \text { is prime }
$$

Proof. $(\Rightarrow)$ We prove that $\widehat{\mathcal{M}}$ satisfies (3) of Proposition 23. Let $u, v \in \operatorname{Fin}(\mathcal{M})$. We claim that

$$
\left\|m_{\widehat{u} \widehat{v}}\right\|=\sup _{\|\widehat{w} \leq 1\|}\|\widehat{u} \widehat{w} \widehat{v}\|={ }^{\circ} \sup _{\|w\| \leq 1}\|u w v\|={ }^{\circ}\left\|m_{u v}\right\|
$$

For the left-to-right inequality, let $\|\widehat{w}\| \leq 1$. Then $\|u w v\| \preccurlyeq\|u z v\|$, for some $\|z\| \leq 1$, where $r \preccurlyeq s$ means $(r \leq s$ or $r \approx s)$. Therefore $\|\widehat{u} \widehat{w} \widehat{v}\|={ }^{\circ}\|u w v\| \leq$ ${ }^{\circ} \sup _{\|z\| \leq 1}\|u z v\|$, from which $\left\|m_{\widehat{u v}}\right\| \leq{ }^{\circ}\left\|m_{u v}\right\|$ follows.

The other inequality follows immediately from the definition of (internal) supremum.

Finally, $\left\|m_{\widehat{u} \widehat{v}}\right\|={ }^{\circ}\left\|m_{u v}\right\|={ }^{\circ}(\|u\|\|v\|)={ }^{\circ}\|u\|{ }^{\circ}\|v\|=\|\widehat{u}\|\|\widehat{v}\|$.

$(\Leftarrow)$ We prove that $\mathcal{M}$ satisfies (2) of Proposition 23. Let $u, v \in \mathcal{M}$ be nonzero elements and let $u_{1}=u /\|u\|, v_{1}=v /\|v\|$. There exists $w \in \operatorname{Fin}(\mathcal{M})$ such that $\widehat{u_{1}} \widehat{w_{0}} \neq 0$. A fortiori $u w v \neq 0$. 


\section{Basics of $K$-theory}

In this section we provide "hull-like" representations of the $K_{0}$ - and the $K_{1}$-groups associated to the nonstandard hull of an internal $C^{*}$-algebra. This is not completely trivial because, in the ultraproduct setting, it is not true, in general, that the $K_{0}$-group of an ultraproduct of $C^{*}$-algebras is the model theoretic ultraproduct of the $K_{0}$-groups of the factors. Similarly for the $K_{1}$-group. We give a hull-like representation of the $K_{1}$-group of a nonstandard hull by looking at the original definition of $K_{1}$ in terms of the notion of suspension.

We begin by recalling that if $\mathcal{M}$ is a concrete $C^{*}$-algebra (i.e. $\mathcal{M} \subseteq \mathcal{B}(\mathcal{H})$ for some Hilbert space $\mathcal{H}$ ) and $n \in \mathbb{N}$, the matrix algebra $M_{n}(\mathcal{M})$ is naturally isomorphic to $\mathcal{B}\left(\mathcal{H}^{n}\right)$ (see [5, II.6.6]).

We fix an internal $C^{*}$-algebra $\mathcal{M} \subseteq \mathcal{B}(\mathcal{H})$. By Transfer of the previous result, we have that, for all $N \in{ }^{*} \mathbb{N}$, the matrix algebra $M_{N}(\mathcal{M})$ is naturally isomorphic to $\mathcal{B}\left(\mathcal{H}^{N}\right)$.

We claim that, for $n \in \mathbb{N}$, the nonstandard hull $\widehat{M_{n}(\mathcal{M})}$ is isomorphic to $M_{n}(\widehat{\mathcal{M}})$. Let $A=\left(a_{i j}\right) \in \operatorname{Fin}\left(M_{n}(\mathcal{M})\right)$. From $\|A\| \geq\left\|a_{i j}\right\|$ (see [5, II.6.6.3]), we get $a_{i j} \in \operatorname{Fin}(\mathcal{M})$ for all $1 \leq i, j \leq n$. Hence $\left(\widehat{a_{i j}}\right) \in M_{n}(\widehat{\mathcal{M}})$. The map

$$
\begin{aligned}
\left.\varphi: \widehat{M_{n}(\mathcal{M}}\right) & \rightarrow M_{n}(\widehat{\mathcal{M}}) \\
\widehat{\left(a_{i j}\right)} & \mapsto\left(\widehat{a_{i j}}\right)
\end{aligned}
$$

is an isomorphism. It is a just a matter of calculation to check the homomorphism requirements. In particular,

$$
\varphi(\widehat{A} \widehat{B})=\varphi(\widehat{A B})=\varphi\left(\left(\sum_{k=1}^{n} a_{i k} b_{k j}\right)^{\wedge}\right)=\left(\sum_{k=1}^{n} \widehat{a_{i k}} \widehat{b_{k j}}\right),
$$

where the rightmost equality follows from finiteness of all $a_{i k}, b_{k j}$ and from $n \in \mathbb{N}$. Therefore $\varphi(\widehat{A} \widehat{B})=\varphi(\widehat{A}) \varphi(\widehat{B})$. The identity $\varphi\left((\widehat{A})^{*}\right)=(\varphi(\widehat{A}))^{*}$ follows from $\left(a_{i j}\right)^{*}=\left(a_{j i}{ }^{*}\right)$.

Moreover

$$
\varphi(\widehat{A})=0 \Rightarrow\left(\widehat{a_{i j}}\right)=(0) \Rightarrow a_{i j} \approx 0 \text { for all } i, j \Rightarrow\|A\| \approx 0,
$$

where the rightmost implication follows from $\|A\| \leq \sum_{i j}\left\|a_{i j}\right\|$. (Notice that the assumption $n \in \mathbb{N}$ is crucial in the previous argument.) Injectivity implies that $\varphi$ is norm-preserving. Surjectivity is trivial.

In the following we shall identify $\left.\widehat{M_{n}(\mathcal{M}}\right)$ with $M_{n}(\widehat{\mathcal{M}})$.

Let $\mathcal{M}$ be an ordinary (internal) $C^{*}$-algebra. Recall that we always work with unital algebras. The idea of associating to $\mathcal{M}$ an abelian group $K_{0}(\mathcal{M})$ marks the beginning of $K$-theory for $C^{*}$-algebras. See [5, V.1.1], from which we borrow the notation. The algebra $M_{\infty}(\mathcal{M})$ of matrices with finitely many (hyperfinitely many, in the internal case) nonzero entries is a not necessarily complete self-adjoint algebra that satisfies the $C^{*}$-axiom, and $V(\mathcal{M})$ is the quotient set of $\operatorname{Proj}\left(M_{\infty}(\mathcal{M})\right)$ with respect to the Murray-von Neumann equivalence relation $\sim$ 
introduced at the beginning of Section 5. We denote by $[p]$ the $\sim$-equivalence class of projection $p$.

It turns out that $(V(\mathcal{M}),+,[0])$ is an abelian semigroup, where + is given by $[p]+[q]=[p+q]$, for $[p],[q]$ equivalence classes of orthogonal projections $p, q$ respectively. (The orthogonality condition is not restrictive. See [5].) Finally, $K_{0}(\mathcal{M})$ is the enveloping group of the semigroup $V(\mathcal{M})$. The definition of $K_{0}(\mathcal{M})$ is more complicated when $\mathcal{M}$ is nonunital, as we shall see later.

From now on we assume that $\mathcal{M}$ is an internal concrete $C^{*}$-algebra of operators. This is not restrictive since what we are going to do extends to arbitrary $C^{*}$-algebras by working with faithful representations of theirs.

For $N \in{ }^{*} \mathbb{N}$, let $\iota_{N}: M_{N}(\mathcal{M}) \rightarrow M_{\infty}(\mathcal{M})$ be the embedding of $M_{N}(\mathcal{M})$ into the upper-left corner of $M_{\infty}(\mathcal{M})$. We shall also denote by $\iota_{n}, n \in \mathbb{N}$, the embedding of $M_{n}(\widehat{\mathcal{M}})$ into the upper-left corner of $M_{\infty}(\widehat{\mathcal{M}})$.

We let

$$
\widehat{V(\mathcal{M})}=\bigcup_{n \in \mathbb{N}^{+}}\left\{\left[\iota_{n}(\widehat{p})\right]: p \in \operatorname{Proj}\left(M_{n}(\mathcal{M})\right)\right\}
$$

Keeping in mind the identification of $\left.\widehat{M_{n}(\mathcal{M}}\right)$ with $M_{n}(\widehat{\mathcal{M}})$ described above, it is easy to check that $\widehat{V(\mathcal{M})}$ is, up to isomorphism, a subsemigroup of $V(\widehat{\mathcal{M}})$.

Actually, let $p \in \operatorname{Proj}\left(M_{\infty}(\widehat{\mathcal{M}})\right)$. Then $p=\iota_{n}(q)$, for some $q \in \operatorname{Proj}\left(M_{n}(\widehat{\mathcal{M}})\right)$. By [24, Theorem 3.22], there exists $r \in \operatorname{Proj}\left(M_{n}(\mathcal{M})\right)$ such that $\widehat{r}=q$. Then $p=\iota_{n}(\widehat{r})$. It follows that $\widehat{V(\mathcal{M})}$ is isomorphic to $V(\widehat{\mathcal{M}})$. Thus the enveloping group of $\widehat{V(\mathcal{M})}$ yields an explicit description of $K_{0}(\widehat{\mathcal{M}})$. Recall that an element of the enveloping group of $\widehat{V(\mathcal{M})}$ can be formally written as $\left[\iota_{n}(\widehat{p})\right]-\left[\iota_{n}(\widehat{q})\right]$, for some $n \in \mathbb{N}$ and some $p, q \in \operatorname{Proj}\left(M_{n}(\mathcal{M})\right)$.

The above definition of $\widehat{V(\mathcal{M})}$ is closely related to the generalized ultraproduct used in [18, Theorem 4.1]. As such, it might be formulated in terms of yet another generalization of the nonstandard hull construction. In [18], the author claims that the latter applies only to get a description of $K_{0}(\widehat{\mathcal{M}})$ and that there seem to be no general way of describing $K_{1}(\widehat{\mathcal{M}})$ as some generalized nonstandard hull. Actually, we prove below that there is a representation of $K_{1}(\widehat{\mathcal{M}})$ which closely follows that of $K_{0}(\widehat{\mathcal{M}})$ just introduced.

Before proving that, we make an aside remark: by means of similar arguments to the above, one can also define a semigroup structure on the set $\left.V_{0}\left(\widehat{M_{\infty}(\mathcal{M}}\right)\right)$ of equivalence classes of projections in $\left.\widehat{M_{\infty}(\mathcal{M}}\right)$. For let $\left.\hat{p} \in \operatorname{Proj}\left(\widehat{M_{\infty}(\mathcal{M}}\right)\right)$. Then there exist $N \in{ }^{*} \mathbb{N}$ and $A \in M_{N}(\mathcal{M})$ such that $A^{2} \approx A ; A^{*} \approx A$ and $p=\iota_{N}(A)$. By [24, Theorem 3.22] we can assume that $A$ is a projection and, consequently, that $p$ is a projection.

Now let $p=\iota_{K}(A)$ and $q=\iota_{N}(B)$ be projections in $M_{\infty}(\mathcal{M})$, for some $K, N \in{ }^{*} \mathbb{N}$ and some $A \in M_{K}(\mathcal{M}), B \in M_{N}(\mathcal{M})$. We define $[\hat{p}]+[\widehat{q}]=[\hat{p}+\widehat{q}]$, where, as mentioned above, we can assume without loss of generality that $\widehat{p}$ and $\widehat{q}$ are orthogonal. It is straightforward to check that + is well defined.

Furthermore $(V(\widehat{\mathcal{M}}),+,[0])$ isomorphically embeds into $\left(V_{0}\left(\widehat{M_{\infty}(\mathcal{M}}\right)\right),+$ $[0])$. Actually, by the above argument, we have that $p \in \operatorname{Proj}\left(M_{\infty}(\widehat{\mathcal{M}})\right)$ can be 
written as $p=\iota_{n}(\widehat{r})$, for some $r \in \operatorname{Proj}\left(M_{n}(\mathcal{M})\right)$. The mapping $\left.[p] \mapsto \widehat{\iota_{n}(r)}\right]$ yields a well defined embedding. In general, $(V(\widehat{\mathcal{M}}),+,[0])$ is a proper subsemigroup of $\left(V_{0}\left(\widehat{M_{\infty}(\mathcal{M})}\right),+,[0]\right)$, as the following example shows. It can be shown that $(V(\mathbb{C}),+,[0]) \simeq(\mathbb{N},+, 0)$. On the other hand, it is not difficult to check that

$$
\widehat{\iota_{M}\left(I_{M}\right)} \sim \widehat{\iota_{N}\left(I_{N}\right)} \Leftrightarrow \iota_{M}\left(I_{M}\right) \sim \iota_{N}\left(I_{N}\right) \Leftrightarrow M=N,
$$

where $I_{K}$ denotes the identity matrix in $M_{K}\left({ }^{*} \mathbb{C}\right)$. The first equivalence above follows from [24, Theorem 3.22(viii)] and from [5, II.3.3.4]. The rightmost equivalence holds by Transfer of the result true in the ordinary setting that, for all $m, n \in \mathbb{N}, \iota_{m}\left(I_{m}\right) \sim \iota_{n}\left(I_{n}\right)$ if and only if $m=n$.

It follows by Saturation that $\left.V_{0}\left(\widehat{M_{\infty}\left({ }^{*} \mathbb{C}\right.}\right)\right)$ has uncountable external cardinality.

Let us deal with $K_{1}(\widehat{\mathcal{M}})$ now. In order to get a hull-like representation of $K_{1}(\widehat{\mathcal{M}})$, where $\mathcal{M}$ is an internal unital $C^{*}$-algebra, we use the original definition of $K_{1}$ based on the notion of suspension.

Let us begin with an ordinary unital $C^{*}$-algebra $\mathcal{M}$ to fix the terminology and the notation. The group $K_{1}(\mathcal{M})$ was originally defined as $K_{0}(S \mathcal{M})$, where the suspension $S \mathcal{M}$ of $\mathcal{M}$ is the $C^{*}$-algebra $\mathcal{C}_{0}(] 0,1[, \mathcal{M})$ of continuous functions $f:] 0,1\left[\rightarrow \mathcal{M}\right.$ such that $\lim _{x \rightarrow 0} f(x)=0=\lim _{x \rightarrow 1} f(x)$. Notice that $S \mathcal{M}$ is nonunital.

In order to retain crucial functorial properties of the construction that hold in the unital case, for instance: half exactness, one considers the unitization $(S \mathcal{M})^{\dagger}$ of $S \mathcal{M}$ (see [5, II.1.2]) and defines $K_{1}(\mathcal{M})$ as a subgroup of $K_{0}\left((S \mathcal{M})^{\dagger}\right)$, as done in $\left[5\right.$, V.1.1.17]. Notice that $(S \mathcal{M})^{\dagger}$ can be identified with $\{f \in \mathcal{C}([0,1], \mathcal{M}): f(0)=$ $\left.f(1) \in \mathbb{C} 1_{\mathcal{M}}\right\}$.

It turns out that $K_{1}(\mathcal{M})$ is isomorphic to the group of formal differences $[p]-[q]$, where

$$
p, q \in \bigcup_{n \in \mathbb{N}^{+}} \iota_{n}\left(\operatorname{Proj}\left(M_{n}\left((S \mathcal{M})^{\dagger}\right)\right)\right)
$$

satisfy the additional conditions $p_{i j}(0)=p_{i j}(1)=q_{i j}(0)=q_{i j}(1) \in \mathbb{C} 1_{\mathcal{M}}$, for all $i, j \in \mathbb{N}$, with the notion of equivalence of formal differences in $K_{0}\left((S \mathcal{M})^{\dagger}\right)$ that comes from the definition of enveloping group.

If we apply the above to the nonstandard hull $\widehat{\mathcal{M}}$ of an internal $C^{*}$-algebra $\mathcal{M}$, we see that, in order to present $K_{1}(\widehat{\mathcal{M}})$ in a nonstandard hull fashion, it suffices to provide a "hull-like" description of $\operatorname{Proj}\left(M_{n}\left((S \widehat{\mathcal{M}})^{\dagger}\right)\right)$.

Our first step in that direction is to provide a suitable description of $(S \widehat{\mathcal{M}})^{\dagger}$.

Let $\mathcal{C}\left({ }^{*}[0,1], \mathcal{M}\right)$ be the internal $C^{*}$-algebra of continuous functions $f: *[0,1] \rightarrow \mathcal{M}$ and let

$$
X=\operatorname{Fin}\left(\left\{f \in \mathcal{C}\left({ }^{*}[0,1], \mathcal{M}\right): f \text { is } S \text {-continuous and } f(0) \approx f(1) \approx{ }^{*} \mathbb{C} 1_{\mathcal{M}}\right\}\right) .
$$

Recall that, for $f, g \in X, f \approx g$ means that $\|f-g\| \approx 0$. On the quotient $\widetilde{X}=X / \approx$, we define the norm $\|f \approx\|={ }^{\circ}\|f\|$, where $f_{\approx}$ denotes the $\approx$-equivalence class of $f \in X$. One can associate to $f \approx$ the continuous function

$$
\tilde{f}:[0,1] \rightarrow \widehat{\mathcal{M}}, \quad{ }^{\circ} x \mapsto \widehat{f(x)}
$$


which satisfies $\widetilde{f}(0)=\widetilde{f}(1) \in \mathbb{C} 1_{\widehat{\mathcal{M}}}$.

Conversely, by the same argument used in the second part of Proposition 11, any $g \in \mathcal{C}([0,1], \widehat{\mathcal{M}})$ with the property that $g(0)=g(1) \in \mathbb{C} 1_{\widehat{\mathcal{M}}}$ can be lifted to an internal $S$-continuous $f:{ }^{*}[0,1] \rightarrow \mathcal{M}$ such that $f(0) \approx f(1) \approx{ }^{*} \mathbb{C} 1_{\mathcal{M}}$. Actually, there exists an internal continuous function $h:{ }^{*}[0,1] \rightarrow \mathcal{M}$ such that $h \approx f:$ pick $N \in{ }^{*} \mathbb{N} \backslash \mathbb{N}$ and let $h$ be the internal piecewise linear function such that $h(i / N)=f(i / N)$ for all $i \in{ }^{*} \mathbb{N}, 0 \leq i \leq N$.

The condition $f \approx h$ is ensured by the already mentioned fact that the supremum of an internal set of infinitesimals is infinitesimal. Consequently, $h$ is $S$-continuous. Moreover $\widetilde{h}=g$.

It is not difficult to check that $\|f \approx\|=\|\tilde{f}\|$, for each $f \in X$. From now on we identify $(S \widehat{\mathcal{M}})^{\dagger}$ with $\widetilde{X}$.

We let $M_{n}(X) \sim$ be the set of equivalence classes

$$
A \approx=\left\{B \in M_{n}(X): B \approx A\right\}, \quad A \in M_{n}(X) .
$$

Notice that if $A \in M_{n}(X)$ then $A \in \operatorname{Fin}\left(M_{n}(\mathcal{C}(*[0,1], \mathcal{M}))\right)$. We define $\|A \approx\|={ }^{\circ}\|A\|$. It is straightforward to check that $M_{n}(X)^{\sim}$ is an involution algebra with respect to the operations defined on representatives of equivalence classes. Moreover it satisfies the $C^{*}$-axiom.

Let $\left(\left(A_{k}\right) \approx\right)_{k \in \mathbb{N}}$ be a Cauchy sequence in $M_{n}(X) \sim$. By completeness of the nonstandard hull $\mathcal{N}$ of $M_{n}(\mathcal{C}(*[0,1], \mathcal{M}))$, we have $\left(\widehat{A_{k}}\right)_{k \in \mathbb{N}} \rightarrow \widehat{A}$, for some $A=\left(a_{i j}\right) \in \operatorname{Fin}\left(M_{n}\left(\mathcal{C}\left({ }^{*}[0,1], \mathcal{M}\right)\right)\right.$. In order to prove that $M_{n}(X) \sim$ is a closed subalgebra of $\mathcal{N}$, it suffices to show that all $a_{i j}, 1 \leq i, j \leq n$, are $S$-continuous and satisfy $a_{i j}(0) \approx a_{i j}(1) \approx{ }^{*} \mathbb{C} 1_{\mathcal{M}}$. As shown at the beginning of this section, we can identify $\widehat{A}$ with $\left(\widehat{a_{i j}}\right)$. Let $1 \leq i, j \leq n$. From $\left(\widehat{A_{k}}\right)_{k \in \mathbb{N}} \rightarrow \widehat{A}$, we get that $\left(\widehat{a_{k i j}}\right)_{k \in \mathbb{N}} \rightarrow \widehat{a_{i j}}$, from which the required properties follow at once.

Hence $M_{n}(X) \sim$ is a $C^{*}$-algebra. Let

$$
\begin{aligned}
\psi: M_{n}(X)^{\sim} & \rightarrow M_{n}(\widetilde{X}) \\
\left(a_{i j}\right) \approx & \mapsto\left(\widetilde{a_{i j}}\right)
\end{aligned}
$$

It is straightforward to check that $\psi$ is a well-defined isomorphism, hence $\left\|\left(a_{i j}\right) \approx\right\|=\left\|\left(\widetilde{a_{i j}}\right)\right\|$. Therefore $M_{n}(X)^{\sim} \simeq M_{n}(\widetilde{X})$. Remember that our aim is to provide a "hull-like" description of $\operatorname{Proj}\left(M_{n}\left((S \mathcal{M})^{\dagger}\right)\right)$. Up to isomorphism, so far we have:

$$
\operatorname{Proj}\left(M_{n}\left((S \mathcal{M})^{\dagger}\right)\right)=\operatorname{Proj}\left(M_{n}(\widetilde{X})\right)=\operatorname{Proj}\left(M_{n}(X)^{\sim}\right)
$$

Finally, we claim that $\left(\operatorname{Proj}\left(M_{n}(X)\right)\right)^{\sim}=\operatorname{Proj}\left(M_{n}(X)^{\sim}\right)$. Concerning the nontrivial inclusion, let $A=\left(a_{i j}\right)$ be such that $[A] \in \operatorname{Proj}\left(M_{n}(X)^{\sim}\right)$. Hence $\left(a_{i j}\right) \approx$ $\left(a_{i j}\right)^{*} \approx\left(a_{i j}\right)^{2}$. Since $M_{n}(X) \subseteq M_{n}(\mathcal{C}(*[0,1], \mathcal{M}))$, it follows from [24, Theorem 3.22] that there exists $\left(b_{i j}\right) \in \operatorname{Proj}\left(M_{n}(\mathcal{C}(*[0,1], \mathcal{M}))\right)$ such that $\left(b_{i j}\right) \approx\left(a_{i j}\right)$. The latter condition implies $\left(b_{i j}\right) \in M_{n}(X)$, by definition of $M_{n}(X)$.

Similarly to what we obtained for $K_{0}(\widehat{\mathcal{M}})$ we thus get a hull-like description of $K_{1}(\widehat{\mathcal{M}})$ : an element of $K_{1}(\widehat{\mathcal{M}})$ can be represented as a formal difference of Murray-von Neumann equivalence classes $\left[\iota_{n}(\widetilde{p})\right]-\left[\iota_{n}(\widetilde{q})\right]$, where $p, q \in \operatorname{Proj}\left(M_{n}(X)\right)$ satisfy the condition $p_{i j}(0) \approx p_{i j}(1) \approx q_{i j}(0) \approx q_{i j}(1) \approx$ ${ }^{*} \mathrm{C} 1_{\mathcal{M}}$, for all $1 \leq i, j \leq n$. 


\section{References}

[1] Albeverio, S.; Fenstad, J.E.; Høegh-Krohn, R.; Lindstrøm, T. Nonstandard Methods in Stochastic Analysis and Mathematical Physics, Academic Press, New York, 1986.

[2] Baratella, S.; $\mathrm{Ng}$, S.A. Some properties of nonstandard hulls of Banach algebras, Bulletin of the Belgian Mathematical Society, 18-1 (2011), 31-38.

[3] Baratella, S.; $\mathrm{Ng}$, S.A. Nonstandard hulls of $\mathrm{C}^{*}$-algebras, http://arxiv.org/abs/0910.5058.

[4] I. Ben Yaacov, A. Berenstein, C.W. Henson and A. Usvyatsov, Model theory for metric structures, in Model theory with applications to algebra and analysis vol II, Z. Chatzidakis et al. ads., London Math. Soc. Lecture Notes Series, no 350, Cambridge University Press (2008), 315-427.

[5] Blackadar B. Operator Algebras, Encyclopaedia of Mathematical Sciences, Vol.122, Springer, New York, 2006.

[6] Chang, C.C.; Keisler H.J. Model theory - 3rd ed., Studies in logic and the foundations of Mathematics vol. 73, North-Holland, Amsterdam, 1990.

[7] Conway, J.B. A course in operator theory. Graduate Studies in Mathematics, 21. American Mathematical Society, Providence, RI, 2000.

[8] Curto R.E.; Hernández G.C. A joint spectral characterization of primeness for C*-algebras. Proc. Am. Math. Soc. 125-11 (1997), 3299-3301.

[9] Dixmier, J. Von Neumann Algebras, North-Holland, Amsterdam, 1969.

[10] Dacunha-Castelle, D.; Krivine, J. L. Applications des ultraproduits à l'étude des espaces et des algèbres de Banach. Studia Math. 41 (1972), 315-334.

[11] Fajardo, S; H.J. Keisler, Neometric spaces. Adv. Math. 118-1 (1996) , 134-175.

[12] Fajardo, S.; .J. Keisler, Existence theorems in probability theory. Adv. Math. 120-2 (1996), 191-257.

[13] Ge, L. and Hadwin, D.; Ultraproducts of $C^{*}$-algebras, in Recent advances in operator theory and related topics, Operator Theory-Advances and applications vol. 127, L. Kérchy et al. eds., Birkhäuser, Basel, 2001.

[14] Hadwin, D.; Li, W. A note on approximate liftings, Oper. Matrices 3-1 (2009), 125-143.

[15] Henson, C.W. Nonstandard hulls of Banach spaces, Israel J. Math. (1976), 108-144.

[16] Hinokuma, T.; Ozawa, M. Conversion from nonstandard matrix algebras to standard factors of type $\mathrm{II}_{1}$, Illinois Journal of Mathematics, 37-1 (1993), 1-13. 
[17] Janssen, G. Restricted ultraproducts of finite von Neumann algebras. Contributions to non-standard analysis (Sympos., Oberwolfach, 1970), 101-114, Studies in Logic and Found. Math. 69, North-Holland, Amsterdam, 1972.

[18] Li, W. On ultraproducts of operator algebras, algebras. Sci. China Ser. A 48-9 (2005), 1284-1295.

[19] Luxemburg, W.A.J. A general theory of monads, in W.A.J. Luxemburg ed., Applications of model theory to algebra, analysis and probability theory, New York (1969), 18-86.

[20] Mathieu, M. Applications of ultraprime Banach algebras in the theory of elementary operators, Ph.D. Thesis (Tubingen, 1986).

[21] McDuff, D. Central sequences and the hyperfinite factor, Proc. London Math. Soc. 21-9 (1970), 443-461.

[22] Megginson, R.E. An introduction to Banach space theory, Springer, New York 1998.

[23] Misonou, Y. On a weakly central operator algebra, Tohoku Math. J. 4-2 (1952), 194-202.

[24] $\mathrm{Ng}$, S.A. Nonstandard methods in functional analysis. Lectures and notes, World Scientific, Singapore, 2010.

[25] Rickart C.E., General theory of Banach algebras, Krieger, New York, 1974.

[26] Sherman, D. Notes on automorphisms of ultrapowers of $\mathrm{II}_{1}$ factors, Studia Math. 195-3 (2009), 201-217.

[27] Somerset, D.W.B. Inner derivations and primal ideals of $C^{*}$-algebras, J. London Math. Soc. 50-2 (1994), 568-580.

[28] Stampfli, J.G. The norm of a derivation, Pacific J. Math. 33 (1970), 737-748.

[29] Takesaki, M. Theory of Operator Algebras I, Springer, New York, 1979.

Dipartimento di Matematica

Università di Trento

I-38123 Povo TN, Italy

email:stefano.baratella@unitn.it 